\title{
KRAV MAGA: ANÁLISE DA PRODUÇÃO CIENTÍFICA
}

\section{REVISÃO BIBLIOMÉTRICA}

ANDRADE NETO, João Batista De ${ }^{1}$

NAVARRO, Antônio Coppi ${ }^{2}$

NAVARRO, Francisco ${ }^{3}$

SALGADO FILHO, Natalino ${ }^{4}$

ANDRADE NETO, João Batista De. Et al. Krav Maga: Análise da produção científica. Revista Científica Multidisciplinar Núcleo do Conhecimento. Ano 05, Ed. 10, Vol. 07, pp. 63-72. Outubro de 2020. ISSN: 2448-0959, Link de acesso: https://www.nucleodoconhecimento.com.br/educacao-fisica/krav-maga

\footnotetext{
${ }^{1}$ Mestrado Acadêmico em Saúde do Adulto; Pós graduado em Fisiologia do Exercício e Biomecânica; Segurança Privada em Safety e Security; Graduado em Licenciatura Plena em Educação Física; Tecnólogo em Segurança Pública e Privada e em Gestão Pública. Mestre em Karatê, Aikidô e Head Coach em Krav Maga.

2 Doutor em Engenharia Biomédica; Mestre em História e Licenciado em Pedagogia.

${ }^{3}$ Doutorado e Mestrado em Ciências; Graduação em Educação Física.

4 Orientador. Doutorado em Medicina (Nefrologia). Mestrado em Medicina (Nefrologia). Especialização em Capacitação Gerencial de Dirigentes Hospitalares. Especialização em Imunopatologia Especial. Especialização em Imunologia. Especialização em Clínica Médica. Especialização em Didática de Nível Superior. Especialização em Residência Médica em Nefrologia. Especialização em Especialização Em Clínica Médica. Aperfeiçoamento em Hipertensão Arterial. Graduação em Medicina.
} 


\section{RESUMO}

O presente estudo objetivou analisar a produção científica de Krav Maga nas principais plataformas acadêmicas de acesso livre no Brasil e no mundo, nos últimos 20 anos (1998-2018). Na revisão sistemática, foram realizadas buscas apenas de artigos originais, utilizando os termos Krav Maga e treinamento físico. Para a análise estatística, foi utilizado o teste qui-quadrado para comparar as proporções. Foram quantificados 362 documentos, dos quais, (98,07\%) encontrados no Portal de Periódicos CAPES/MEC. Quanto à qualificação, foram encontrados: (21) Artigos científicos; (07) resenhas de livros; (268) artigos de jornais não científicos; (13) cinema; (24) revistas; (01) biografia; (13) moda, 11 (vendas); (05) agricultura e (01) etiologia. Esses resultados indicam a necessidade emergente de investigações acerca do tema Krav Maga, principalmente sobre os efeitos de seu treinamento físico, bem com a aproximação crítico-dialógica e da práxis educacional do ponto de vista acadêmico e profissional por parte dos múltiplos agentes que dinamizam e reconfiguram as manifestações corporais e de movimento desta modalidade de luta.

Palavras-chave: Krav Maga, treinamento físico, fisiologia do exercício, lutas, artes marciais.

\section{INTRODUÇÃO}

O Krav Maga (KM) é uma das modalidades de lutas que mais cresce no Brasil e no mundo atualmente. Desenvolvida no Estado de Israel na década de 40, utiliza-se de golpes traumáticos, pressões para derrubar ou dominar o oponente, torções e imobilizações, visando a neutralizar o ataque e o agressor. Esse método de exercício corporal para defesa pessoal foi desenvolvido por IMI Lichtenfeld, com bases técnicas no Jiu Jitsu, Boxe, Wrestling e Judô.

No Brasil, o KM chegou nos anos 90, apresentando-se como arte de defesa pessoal, ganhando rapidamente reconhecimento nos mais variados espaços e instituições sociais como atividade física, lazer, esporte, caminho filosófico, técnica marcial ou como defesa pessoal, principalmente no âmbito das Forças Armadas e Órgãos de 
Segurança Pública (LICHTENSTEIN 2016; FARKASH et al., 2017; ANDRADE NETO, 2019).

De forma holística, é cabível o emprego da expressão "Krav Maga - a defesa pessoal do Exército israelense", pois direciona a um entendimento diversificado de representações e significados, o que por sua vez, Ihe confere uma dimensão polissêmica. Como exemplificação, temos as noções de arte marcial para defesa pessoal; sistema eclético de defesa pessoal; arte para defesa pessoal; sistema operacional de defesa pessoal militar, dentre e outros.

De acordo com Andrade Neto (2019), o Krav Maga foi idealizado e desenvolvido metodologicamente dentro das forças de resistência e defesa que lutavam pela independência do Estado de Israel e para preparar a sua população no enfrentamento da violência que lá existia. Assim como todas as Lutas e Artes Marciais preexistentes, nasceu e se desenvolveu da necessidade de sobrevivência e independência de um povo. E com a criação do Estado de Israel em 1948, teve início seu processo de expansão mundial, mais acentuadamente a partir da década de 70, após a realização do primeiro Curso de Instrutores de Krav Maga pelo Colégio Acadêmico de Wingate.

Contudo, apesar de sua popularidade, raros são os estudos científicos desenvolvidos sobre o tema Krav Maga e suas formas de treinamento físico. $O$ único trabalho acadêmico encontrado, que faz alusão às origens da modalidade como arte marcial para a defesa pessoal, segundo Mayers e Tuchman (2009), foi publicado em 2006 pela Universidade Judaica de Jerusalém, em Israel. Nesse estudo, os autores afirmam que "não existe" nenhuma análise que explique por que o Krav Maga foi criado justamente em Israel e quais os métodos de treinamento. Essa foi, então, a primeira tentativa de se explicar cronologicamente o surgimento e evolução da modalidade. Tal pesquisa de cunho bibliográfico se concentrou nos 40 anos iniciais da modalidade (de 1967 a 2007) e nada foi encontrado.

Essa ausência de referências científicas induz a uma reprodução das técnicas e métodos de treinamentos do Krav Maga, semelhantes às demais modalidades de Lutas e Artes Marciais em todo o mundo, as quais são ensinadas e transmitidas por 
instrutores/professores aos seus alunos, majoritariamente baseados na mesma metodologia em que foram formados. E estes, por sua vez, reproduzem de maneira análoga o conhecimento obtido para os próximos alunos, o que Drigo (2011) chama de "método artesanal", métodos de ensino na base de erros e tentativas, ou seja, de forma empírica.

$\mathrm{Na}$ perspectiva das publicações sobre os métodos de treinamento físico e os efeitos fisiológicos produzidos por sua prática, o presente estudo objetivou analisar a produção científica de Krav Maga nas principais plataformas de pesquisa acadêmicas de livre acesso no Brasil e no mundo, nos últimos 20 anos.

\section{MATERIAIS E MÉTODOS}

A revisão sistemática da literatura foi realizada nas principais plataformas de pesquisa utilizadas no Brasil e no mundo. Foram adotadas como critério de seleção, as bibliotecas virtuais que apresentassem artigos científicos de livre acesso, texto completo e que tivessem publicações disponíveis nos últimos 20 anos (1998-2018). Não foi feito busca por teses ou dissertações, trabalhos de conclusão de curso manuais, face à disponibilidade de acesso às bases de pesquisa selecionadas. Ênfase foi dada a pesquisas com implicações diretas para a intervenção, especialmente no que diz respeito aos efeitos fisiológicos e preparação física. A palavra de busca usada inicialmente na pesquisa foi "Krav Maga".

Foram utilizadas como fontes de pesquisa 6 (seis) plataformas com os seguintes endereços eletrônicos:

http://www.periodicos.capes.gov.br/; http://www.scielo.org/php/index.php, http://www.scielo.br/, https://dialnet.unirioja.es/, https://www.redib.org/, http://bases.bireme.br. A busca foi realizada no período de julho a dezembro de 2018. Ressalta-se que apenas artigos científicos originais foram analisados.

A análise estatística dos dados foi composta pelo teste qui-quadrado para comparar proporções e analisar possíveis associações entre as variáveis. Todas as análises 
foram efetuadas em software estatístico BioEstat, versão 5.3, bem como o nível de significância foi pré-fixado em $p<0,05$.

\section{RESULTADOS}

Tabela 1 - quantificação dos documentos encontrados

\begin{tabular}{|l|l|l|l|l|l|l|}
\hline $\begin{array}{l}\text { Periódicos } \\
\text { Capes/MEC }\end{array}$ & SciElo.org & SciElo.br & Dialnet & Redib & Bireme & Total \\
\hline $\mathbf{3 5 7}$ & 0 & 0 & 2 & 1 & 2 & 362 \\
\hline
\end{tabular}

Fonte: elaboração própria.

$\mathrm{Na}$ Tabela 1, pode-se verificar um número significativo de 362 publicações encontradas nas bibliotecas virtuais pesquisadas com a palavra Krav Maga. Deste total, $98,07 \%$ no Portal de Periódicos CAPES/MEC, os demais se distribuem nas bibliotecas scielo.br e scielo.org ebireme.br que corresponde $0,96 \%$ dos achados, totalizando $1,93 \%$. No entanto, estas publicações se duplicam com os já listados.

Quadro 2 - qualificação das publicações encontradas por assuntos relacionados

\begin{tabular}{|l|l|l|l|l|l|l|l|l|l|}
\hline $\begin{array}{l}\text { Artigos } \\
\text { Científi } \\
\text { cos }\end{array}$ & $\begin{array}{l}\text { Resen } \\
\text { has de } \\
\text { Livros }\end{array}$ & $\begin{array}{l}\text { Artig } \\
\text { os }\end{array}$ & $\begin{array}{l}\text { Cine } \\
\text { ma }\end{array}$ & $\begin{array}{l}\text { Revis } \\
\text { tas }\end{array}$ & $\begin{array}{l}\text { Biogr } \\
\text { afia }\end{array}$ & $\begin{array}{l}\text { Mo } \\
\text { da }\end{array}$ & $\begin{array}{l}\text { Vend } \\
\text { as }\end{array}$ & $\begin{array}{l}\text { Agricul } \\
\text { tura }\end{array}$ & $\begin{array}{l}\text { Etiolo } \\
\text { gia }\end{array}$ \\
\hline 21 & 07 & 268 & 13 & 24 & 01 & 13 & 11 & 05 & 01 \\
\hline
\end{tabular}

Fonte: elaboração própria.

No quadro 2, a classificação das publicações com a temática Krav Maga, realizada segundo a estruturação indireta de assuntos proposta pela CAPES/2017. Foram encontrados os seguintes resultados: Artigos científicos (21); resenhas de livros (07); 
artigos de periódicos (268); cinema (13); revistas (24); biografia (01); moda (13), vendas (11); agricultura (05) e etiologia (01).

Quadro 3 - Classificação dos artigos científicos.

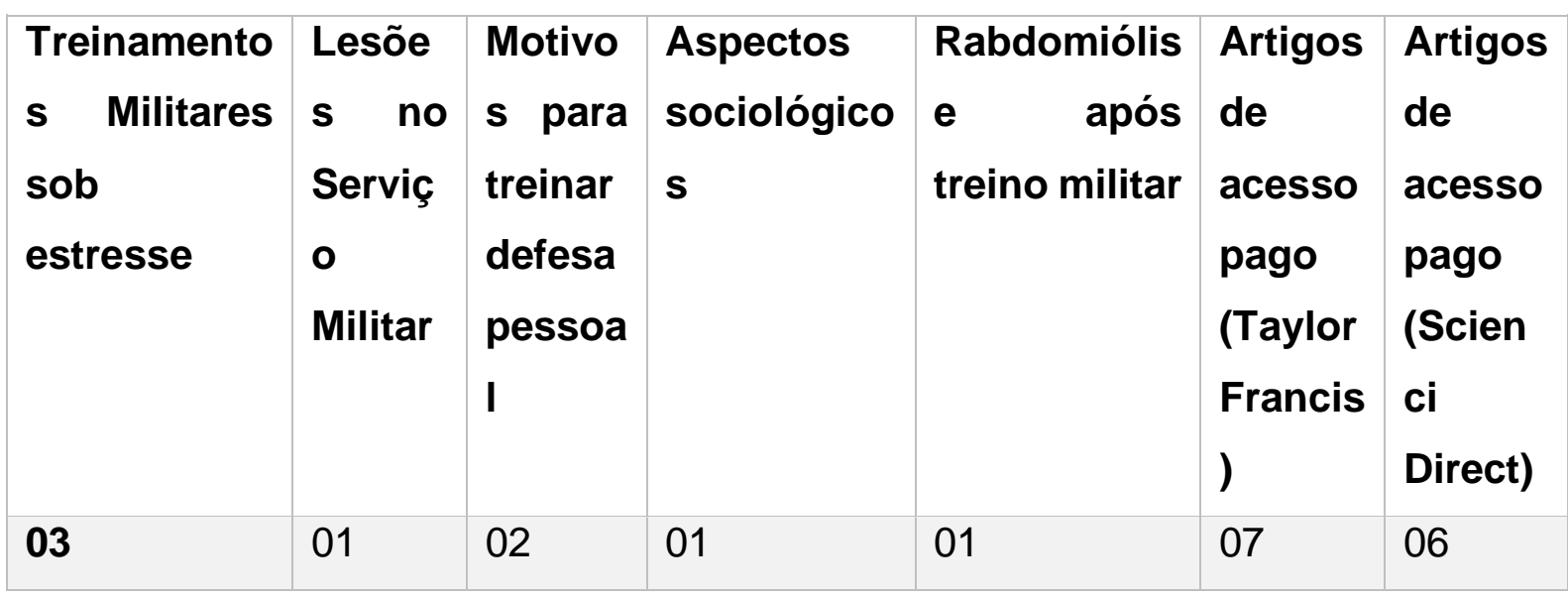

Fonte: elaboração própria.

No quadro 3, dos (21) artigos encontrados que direcionam a estudos científicos com a temática Krav Maga, foram divididos em diferentes áreas do conhecimento, sendo: treinamentos militares sob estresse (03); lesões no serviço militar (01); motivos para treinar defesa pessoal (02); aspectos sociológicos (01); rabdomiólise após treino militar (01) e artigos pagos nos periódicos Taylor Francis (07) e Scienci Direct (06).

\section{DISCUSSÃO}

Os resultados obtidos apontam para um baixo e quase inexistente número de artigos científicos direcionados às atividades de Krav Maga que possam nortear suas metodologias, técnicas e táticas usadas no ensino-aprendizagem ou prescrição de treinamento físico para uso habitual.

Notadamente, destacam-se nos documentos encontrados no presente estudo publicações científicas com ênfase ao treinamento físico-militar e no tratamento de lesões causadas por essas atividades. Para Farkash et al., (2017), o Krav Maga é um sistema de combate corpo a corpo israelense único, criado e projetado para ensinar aos soldados a autodefesa em situações reais. E, apesar de sua ampla popularidade 
e uso civil, suas origens, história e evolução não foram examinadas seriamente na literatura acadêmica, nem seu papel principal na promoção do uso de reações reflexas em combate (GUY MOR, 2018).

Ressalta-se, aqui, o consenso na literatura de que existe uma lacuna entre a práxis das lutas, artes marciais e modalidades esportivas de combate (L/AM/MEC). Neste caso específico o Krav Maga e a produção científica, apesar de sua crescente demanda social.

Correia e Franchini (2010) analisaram a produção acadêmica em lutas, artes marciais e esportes de combate nas principais revistas acadêmicas de circulação nacional da área de Educação Física, após o estabelecimento do sistema Conselho Federal de Educação Física no Brasil. Este, que se constituiu até então o mais pormenorizado estudo acerca do tema, foi realizado no período de 1998 a 2008. No levantamento, foi constatado o predomínio dos estudos conduzidos na área de Biodinâmica (40\%), seguidos pelos Estudos Socioculturais do Movimento Humano (32\%) e Comportamento Motor (8\%). Os estudos aplicados foram divididos em: Pedagogia do Movimento Humano (10,7\%), Treinamento Esportivo (8\%), Administração Esportiva (1,3\%) e Adaptação do Movimento Humano (nenhum artigo produzido). Como resultado os autores apontam uma carência de publicações sobre essas atividades, especialmente as de caráter aplicado e, ainda, a necessidade de investimentos em pesquisas inter e multidisciplinares sobre essa temática.

Logo após, Franchini e Del Vecchio (2011) apresentaram o estado da arte dos estudos sobre as modalidades esportivas de combate (MEC), objetivando destacar a relevância destas modalidades, tanto do ponto de vista histórico, quanto em relação à sua representatividade em competições internacionais e as áreas mais comuns de atuação do profissional de esporte, bem como, as iniciativas de organização de eventos, publicações, grupos de estudos e instituições científicas direcionadas às modalidades esportivas de combate.

Os autores notaram um aumento das pesquisas sobre as MEC, bem como os resultados positivos em competições esportivas no Brasil. Por outro lado, raros foram 
os financiamentos encontrados, principalmente no que se referia aos efeitos dos exercícios físicos para alta performance em atletas de MEC.

Em outro estudo, Jacomin et al., (2013), com um modelo de revisão sistemática da literatura nacional, analisaram a produção de artigos científicos na área de lutas, através da base de dados Scientific Electronic Library Online (SciELO), entre os anos de 1998 a 2013. No estudo, foram encontrados 24 trabalhos científicos publicados em dez periódicos. Os autores observaram um maior número de estudos relacionados ao Judô quando comparados às outras modalidades (Judô: $58,7 \%$, outras: $41,3 \%$ ) que, os estudos encontrados enfocam mais o esporte (esporte: $79,5 \%$, saúde: $8,2 \%$, educação: $4,1 \%$ e outros: $8,2 \%$. Também apontam a necessidade de mais investigações acerca do tema artes marciais, bem como mais trabalhos com o sexo feminino, com idosos, crianças e adolescentes.

Também foi percebido durante o I Colóquio de Lutas, Artes Marciais e Esportes de Combate da FEF - UNICAMP realizado em 2013, pelo Grupo de Estudo e Pesquisa em Lutas da Faculdade de Educação Física da Universidade Estadual de Campinas, sobre a produção acadêmica em lutas no Brasil, que as pesquisas sobre o tema apresentam fragilidades metodológicas, principalmente no rigor científico e consistência metodológica, as quais dever ser levadas em consideração para a qualificação destas produções.

Em outro estudo envolvendo a temática, Antunes (2016) faz reflexões e possíveis encaminhamentos afetos as lacunas e escassez de produções acadêmicas sobre L/AM/MEC atual e dos últimos anos. O autor destaca, em especial ausência de estudos que tratam da intervenção, do ensino e da aprendizagem e estudos com grupos específicos de crianças, mulheres, idosos e pessoas com deficiência.

Para o autor, estes grupos se constituem novos personagens oriundos de novos cenários emergentes na atualidade. Abrindo assim, possibilidades de novas formas de práticas, vinculadas às subjetividades de seus praticantes. E ainda, sinaliza a necessidade de abertura de novos modelos de intervenção e criação de novos grupos 
de pesquisa capazes de dialogar, sobre as mesmas temáticas ou adjacente sem criarem barreiras, dentro de seus próprios grupos de pesquisa.

Após considerar as informações fornecidas pela literatura e a comparando-as com os achados neste estudo, percebe-se, de forma geral, a iminente necessidade de investigação sobre intervenção, do ensino e da aprendizagem e dos efeitos fisiológicos provocados pelo treinamento técnico, tático e físico do Krav Maga. Nos poucos artigos encontrados, a maior parte é relacionada as atividades de ensino em organizações militares e órgãos de segurança com metodologia específica. Nota-se, então, que, desde a origem até os dias atuais, uma constante no modelo de ensino militarizado, face a sua origem e adoção nos maiores e mais conhecidos times táticos operacionais do mundo (FARKASH et al, 2017; GUY MOR, 2018; ANDRADE NETO, 2019).

Para Antunes et al. (2016) existe, neste caso, um distanciamento entre pesquisadores e professores/instrutores, uma vez que os objetos de estudo, as linguagens, as condições de elaboração do conhecimento e as especificidades profissionais são distintos, levando para uma dificuldade na interlocução acadêmica e profissional. Exemplificando esse contexto, o autor cita a situação expressa por aqueles que atuam na Educação Básica, por não saberem o que os pesquisadores produzem e estes não sabem como os professores de Educação Básica atuam.

Andrade Neto (2019) assinala que o treinamento de Krav Maga em todo mundo atualmente é realizado às escuras, pois a ausência de referências de pesquisas acadêmicas direcionadas ao ensino, a prescrição dos treinamentos, as metodologias utilizadas, a estrutura técnica e tática de treinamento são feitas por analogia a outras modalidades de Lutas /Artes Marciais/Modalidades Esportivas de Combate (L/AM/MEC), desvirtuando a essência da modalidade.

Para Franchini e Del Vecchio (2011) boas estratégias neste sentido poderiam englobar promoção de cursos de formação, aperfeiçoamento profissional e maior quantidade de relações entre universidades e entidades representativas, o que na prática não ocorre. Os cursos hoje realizados de Krav Maga são de entidades 
particulares e independentes, muitos sem fundamentação técnico-científica, realizados apenas com experiências de seus instrutores em outras modalidades ou esportes.

Nas últimas décadas, um grande número de estudos sobre os efeitos benéficos da prática de L/AM/MEC como exercício físico sobre o organismo vem sendo documentado e recomendado em indivíduos adultos saudáveis, em diversos marcadores fisiológicos, seja para a melhoria da aptidão física relacionada à saúde em geral (SCHWARTZ, 2011; MACÊDO et al., 2016) e desenvolvimento das habilidades esportivas (RASSOVSKY et al., 2019) seja sobre aspectos pedagógicos e psicossociais (GOMES, 2014; GONÇALVES, 2015). Contudo, a falta de estudos investigando os efeitos das atividades no Krav Maga é, ainda, um problema a ser sanado pela comunidade científica.

\section{CONCLUSÃO}

De maneira geral, qualquer tentativa de se obter resultados satisfatórios baseando-se apenas em pesquisa acadêmica sobre o Krav Maga é bastante restritiva em termos de publicações acadêmicas. Especialmente estudos sobre a introdução, desenvolvimento e métodos de treinamento do Krav Maga no Brasil e no mundo.

A prática baseada em evidências é uma abordagem que possibilita a melhoria da qualidade do ensino e prescrição de um treinamento adequado a alunos e clientes. Essa abordagem envolve a definição de um problema, a busca e avaliação crítica das evidências disponíveis (pesquisas), implementação das evidências na prática e avaliação dos resultados obtidos.

No presente artigo, apresentamos a revisão sistemática que consistirá em um ponto inicial e auxiliará as futuras pesquisas, pois esse recurso possibilita a síntese das pesquisas disponíveis, relativas a um determinado problema, com a finalidade de direcionar a prática fundamentada em conhecimento científico. Pois os estímulos e adaptações funcionais e estruturais produzidos pela prática regular de exercícios físicos sobre o organismo são uma estratégia preventiva, primária, atrativa e eficaz de 
combate ao sedentarismo, contribuindo de maneira significativa para a manutenção da aptidão física e melhoria da saúde e de outros fatores.

O Krav Maga ou defesa pessoal israelense, como apresentado, é uma modalidade de luta notadamente recente, que ganha cada vez mais importância no cenário contemporâneo, seja em ambientes formais e não formais de ensino ou em órgãos de segurança. Contudo, carente de debates e reflexões científicas, pois sua prática se constitui como atividade física e, como tal, deve ser realizada prioritariamente para o aprimoramento da saúde nas dimensões mais amplas possíveis.

Sendo assim, de forma retórica sugerimos a aproximação crítico-dialógica e da práxis educacional do ponto de vista acadêmico e profissional por parte dos múltiplos agentes dos saberes que dinamizam e reconfiguram as manifestações corporais e de movimento, mais especificamente as relacionadas ao ensino do Krav Maga.

Espera-se que o presente trabalho contribua para esse fortalecimento das áreas de ensino técnico-metodológico e de treinamento físico e sirva de referência para novos estudos e questionamentos. Acredita-se que a tendência seja melhorar a qualidade da pesquisa científica e com isso se consiga uma identidade mais homogênea da modalidade, tanto no âmbito acadêmico quanto no mercado profissional.

\section{REFERÊNCIAS}

ANTUNES, M.M. A Produção Acadêmica em Lutas, Artes Marciais e Esportes de Combate: Reflexões e Possíveis Encaminhamentos. Revista Brasileira de Prescrição e Fisiologia do Exercício, São Paulo. v.11. n.63. p. 921-924. Edição Especial. 2016. ISSN 1981-9900.

ANTUNES, M.M.; ALMEIDA, J.J.G. Artes Marciais, lutas e esportes de combate na perspectiva da Educação Física reflexões e possibilidade. CRV. Curitiba. 2016.

ANDRADE NETO, J.B. Efeitos Fisiológicos do Treinamento Físico de Krav Maga nas variáveis: hemodinâmica, metabólica, hidratação, neuromuscular, hormonal e sono. 
2019. 106f. Dissertação (Mestrado em Saúde do Adulto) Centro de Ciências Biológicas - Universidade Federal do Maranhão - UFMA, São Luís, 2019.

BRASIL. Portal de Periódicos da Coordenação de Aperfeiçoamento de Pessoal de Nível Superior/CAPES. Disponível em: http://www.periodicos.capes.gov.br/. Acesso em 31 Dez 2018.

DRIGO, A, J. Artes marciais, formação profissional e escolas de ofício: Análise documental do judô brasileiro. Motricidade. 2011, vol. 7, n. 4, pp. 49-62

FARKASH, U., DREYFUSS, D., FUNK, S. Prevalence and Patterns of Injury Sustained During Military Hand-to-Hand Combat Training Krav-Maga. AMSUS - Association of Military Surgeons of the U.S. MILITARY MEDICINE, Vol. 182, 2017.

FRANCINI, E.; DEL VECCHIO, F.B. Estudos em modalidades esportivas de combate: estado da arte. Revista brasileira de Educação Física e Esporte. São Paulo. Vol. 25 Núm. esp. p.67-81. 2011.

GOMES, MARIANA SIMÕES PIMENTEL. O ensino do saber lutar na universidade: estudo da didática clínica nas Lutas e Esportes de Combate. 204f. Tese (doutorado em Educação Física) Faculdade de Educação Física. Universidade Estadual de Campinas, Campinas, 2014.

GONÇALVES ECA, SILVA DAS, NUNES HEG. Prevalence and factors associated with low aerobic performance levels in adolescents: a systematic review. Curr Pediatr Rev 2015;11(1):65-70. https://doi.org/10.5007/1980-0037.2018v20n4p446

LICHTENSTEIN, K. Krav Maga: a filosofia da defesa israelense. $2^{a}$ edição. Imago. Rio de Janeiro. 2016.

MAYERS, G.; TUCHMAN, J. Gênesis: a história do Krav Maga. Editora Livre Expressão. Rio de Janeiro. 2009. 
MACEDO, EVS.; CAVAlCANTE, JR.; LIMA, LA.; et al. Níveis de força em adolescentes de diferentes estágios maturacionais. Revista Brasileira de Fisiologia do Exercício. São Paulo, v.15, p.77-80, 2016. Número 2.

RASSOVSKY, Y; HARWOOD, A, SHARON, O.Z.; FELDMAN, R.: Martial Arts Increase Oxytocin Production Scientificreports Natureresearch. (2019) 9:12980 https://doi.org/10.1038/s41598-019-49620-0

SCHWART, JULIANO. Aptidão física relacionada à saúde e qualidade de vida de praticantes de lutas, artes marciais e modalidades de combate da cidade de São Paulo. 2011. Dissertação (Mestrado em Biodinâmica do Movimento Humana) - Escola de Educação Física e Esporte, Universidade de São Paulo, SP, 2011. Doi:10.11606/D.39.2011.tde-170222012-085028.

Enviado: Setembro, 2020.

Aprovado: Outubro, 2020. 